\title{
Separation of Chiral Magnetic Effects From Flow Effects in U+U Collisions
}

\section{Sandeep Chatterjee*}

Variable Energy Cyclotron Centre, Kolkata

E-mail: sandeepc@vecc.gov. in

\section{Prithwish Tribedy}

Variable Energy Cyclotron Centre, Kolkata

E-mail: ptribedyavecc.gov.in

\begin{abstract}
One of the major challenges in the search of chiral magnetic effect (CME) in heavy ion collisions is to reduce the background contribution arising from elliptic flow. This is mainly because conventional efforts to increase the CME also result in higher flow effects. So far, attempts towards disentangling the two effects have been restricted to ultra-central U+U events where CME is expected to vanish even for events with non-zero elliptic flow. In this work we propose a novel method to systematically untangle CME from flow effects over a wide range of centralities. We show that the geometry of the Uranium nucleus gives rise to unique collision configurations which can effectively separate out CMEs from flow effects- enhancing CME and at the same time reducing flow and vice versa. We demonstrate that the large spectator asymmetry that these events generate can be used to trigger them.
\end{abstract}

7th International Conference on Physics and Astrophysics of Quark Gluon Plasma

1-5 February, 2015

Kolkata, India

\footnotetext{
* Speaker.
} 
The initial stages of a heavy ion collision (HIC) experiment offers the unique opportunity to study local $P$ and $C P$ odd domains in the presence of strong electromagnetic field, giving rise to a plethora of novel transport phenomena like the chiral magnetic effect (CME), chiral separation effect (CSE) etc. For detailed discussions on these anomalous transports and reference list we refer the readers to the following recent review articles $[1,2,3]$. In this proceeding, we will restrict our discussion to CME and elucidate a way of removing the dominant background contribution due to flow on signal of CME.

The external magnetic field breaks the isotropy in space which manifests in the preferential emission of the charged particles along the direction of the external magnetic field with the (un)like sign charges (anti-)aligned [4]. An observable that has been proposed to measure such final state charge configuration is [5]

$$
\gamma^{a b}=\left\langle\cos \left(\phi^{a}+\phi^{b}-2 \psi_{R P}\right)\right\rangle
$$

Here $\psi_{R P}$ is the reaction plane angle, $\phi$ is the azimuthal angle of the particle and $a, b= \pm$, is its charge state. The above quantity even though removes background contribution due to non-flow effects like resonance decays, still receive contribution from flow effects. Any attempt to reduce flow effects by going towards more central collisions also lessen the number of spectators and thus reduction in the magnetic field. This eventually leads to fall in the CME signal. Hence, in spite of having observed non-zero $\gamma^{a b}$ in $\mathrm{Au}+\mathrm{Au}, \mathrm{Cu}+\mathrm{Cu}$ and $\mathrm{Pb}+\mathrm{Pb}$ collisions $[6,7,8,9]$, the jury is still out on on CME [10].

There have been several suggestions on disentangling the CME from flow in $\mathrm{Au}+\mathrm{Au}$ and $\mathrm{U}+\mathrm{U}$ collisions $[11,12]$. Here we use to our advantage the deformed geometry of the Uranium nucleus to separate CME like signal from flow effects across a wide range of centralities in $\mathrm{U}+\mathrm{U}$ collisions [13]. We perform Monte Carlo Glauber model (MCGM) simulations to generate the initial magnetic field and participant eccentricities created in $\mathrm{U}+\mathrm{U}$ collisions and demonstrate how the asymmetry in the left $(\mathrm{L})$ and right $(\mathrm{R})$ going spectator neutrons $(|L-R|)$ provide us with a tuning parameter to separate flow from $\mathrm{CME}$ in $\mathrm{U}+\mathrm{U}$ collisions.

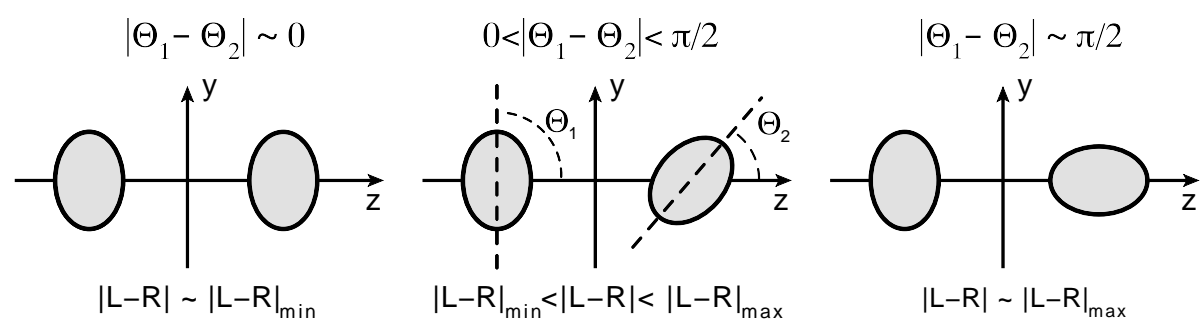

Figure 1: Geometries of $\mathrm{U}+\mathrm{U}$ collisions that generate higher spectator neutron asymmetry $|L-R|$ due to increase in the angle between the major axes of two nuclei at a fixed impact parameter. The collision direction is chosen along the $\mathrm{z}$-axis [13].

The details of the two component MCGM used in this study is given in Ref. [13]. Fig. 1 illustrates the strategy adopted in this work to untange CME from flow. The spectator asymmetry $|L-R|$ in full overlap $\mathrm{U}+\mathrm{U}$ collisions gets contribution only from quantum fluctuations of the nucleon positions. Thus for such configurations, we have a minimal value say $|L-R|_{\min }$ for the spectator asymmetry. Also we should keep in mind that due to the prolate shape, these events have 
large anisotropy in the initial energy density deposited. As we go towards event bins with larger spectator asymmetry, it is clear from Fig. 1 that the anisotropy reduces monotonically. This is how by tuning $|L-R|$ it is possible to regulate the initial eccentricity of the event. Further, the full overlap collision configuration correspond to the case with minimal spectators and hence minimum CME. On the other hand those with large $|L-R|$ are expected to reveal larger CME signal by virtue of having larger number of spectators. Thus, the prolate geometry of the Uranium nucleus provides us with this unique probe, $|L-R|$, tuning which results in anti-correlation between flow and CMEin the process separating the two phenomena.

We will now look at some of the results that we have obtained from our MCGM simulations. As a proxy for $\gamma^{a b}$ we use the frequently used correlation of the $B$ field with the reaction plane $\Psi_{R P}$, $\gamma^{B}[14]$ defined as

$$
\gamma^{B}=e^{2} B^{2} \cos \left(2\left(\Psi_{B}-\Psi_{R P}\right)\right)
$$

Here $\Psi_{B}$ is the angle of the resultant $B$-field vector at the point of observation due to all protons. This quantity is similar to Eq.1 of $\gamma^{a b}$ with $\phi_{a}+\phi_{b}$ replaced by $2 \Psi_{B}$. In this work we estimate the values of $B$ and $\Psi_{B}$ at the time of collision using the expression of Lienard-Wiechert potentials [15] at the central point of the participant zone defined by the average weighted positions of the participants. In experiments $\Psi_{R P}$ is estimated either from the orientation of the emitted particles $\left(\Psi_{E P}\right)$ or from the directed flow of the spectator neutrons using the signals from ZDCs $\left(\Psi_{Z D C}\right)[8,16]$. Accordingly, in our model calculation we estimate $\Psi_{R P}$ in two different approaches. Firstly, we use the positions of the participants to define the orientation of the participant plane in the following way

$$
\varepsilon_{2} e^{i 2 \Psi_{2}^{P P}}=\frac{\sum_{p} r_{p}^{2} e^{i 2 \phi_{p}}}{\sum_{p} r_{p}^{2}},
$$

where the sum is performed over the positions of participant nucleons (assumed to be deltafunctions) at positions $\left(r_{p}, \phi_{p}\right)$ and $\varepsilon_{2}$ is the ellipticity of the participant zone. Here $\Psi_{2}^{P P}$ denotes the second order participant plane, a measure of the direction of the initial state spatial anisotropy. In order to serve as a proxy for $\Psi_{E P}$ that measures the orientation of the final state momentum anisotropy, $\Psi_{2}^{P P}$ is rotated by $\pi / 2$ as per convention. Secondly, we also estimate the spectator plane $\Psi_{2}^{S P}$ using the positions of spectator neutrons.

We now present our proposal to disentangle $\gamma^{a b}$ from $v_{2}$ in experiments:

- Firstly, measure the event averaged $v_{2}$ and $\gamma^{a b}$ in different centrality bins with $|L-R|=0$. This will provide the correlation plot of $\gamma^{a b}$ vs $v_{2}$ by varying the centrality alone as shown by the dotted line (and solid symbols) in Fig. 2 where $\gamma^{B}$ proportionally increases with $\varepsilon_{2}$ for different centrality bins.

- Secondly, every centrality bin is divided into further bins of different $|L-R|$ and the event averaged $v_{2}$ and $\gamma^{a b}$ for each such $|L-R|$ bin is measuerd and the same correlation plot is prepared as shown by solid lines (and open symbols) for four different centralities in Fig. 2. Thus introduction of the $|L-R|$ parameter allows us to tune $\varepsilon_{2}$ in a given centrality class.

In Fig. 3 we plot the variation of $\varepsilon_{2}$ and $\gamma^{B}$ scaled by their mean values in a given centrality bin with respect to $|L-R|$. For both centralities, $\varepsilon_{2}$ monotonically decreases with increasing $|L-R|$ 


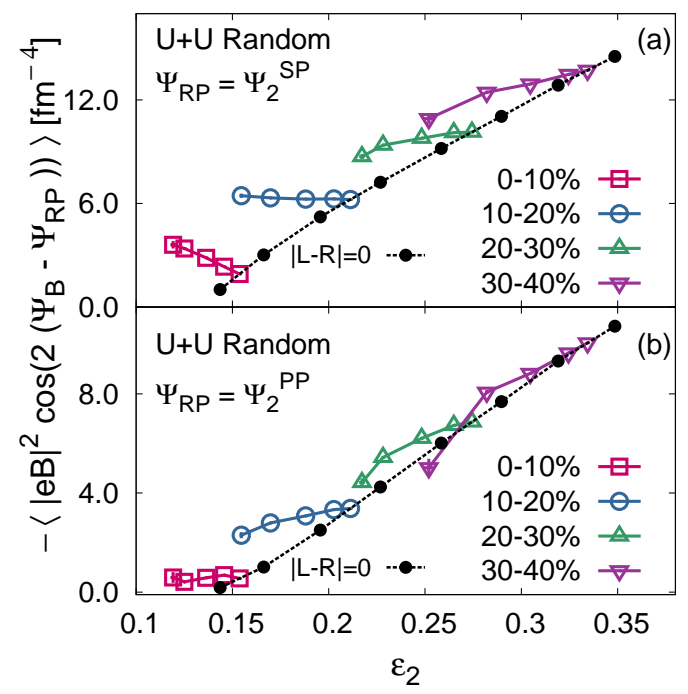

Figure 2: (Color online) The correlator $\gamma^{B}$ at the centre of the participant zone vs $\varepsilon_{2}$ for different centrality and spectator asymmetry bins. Top: $\gamma^{B}$ measured with respect to the 2 nd spectator plane. Bottom: $\gamma^{B}$ measured with respect to the 2 nd participant plane. Bins with same $\varepsilon_{2}$ is observed to have multiple $\gamma^{B}$ and vice-versa [13].

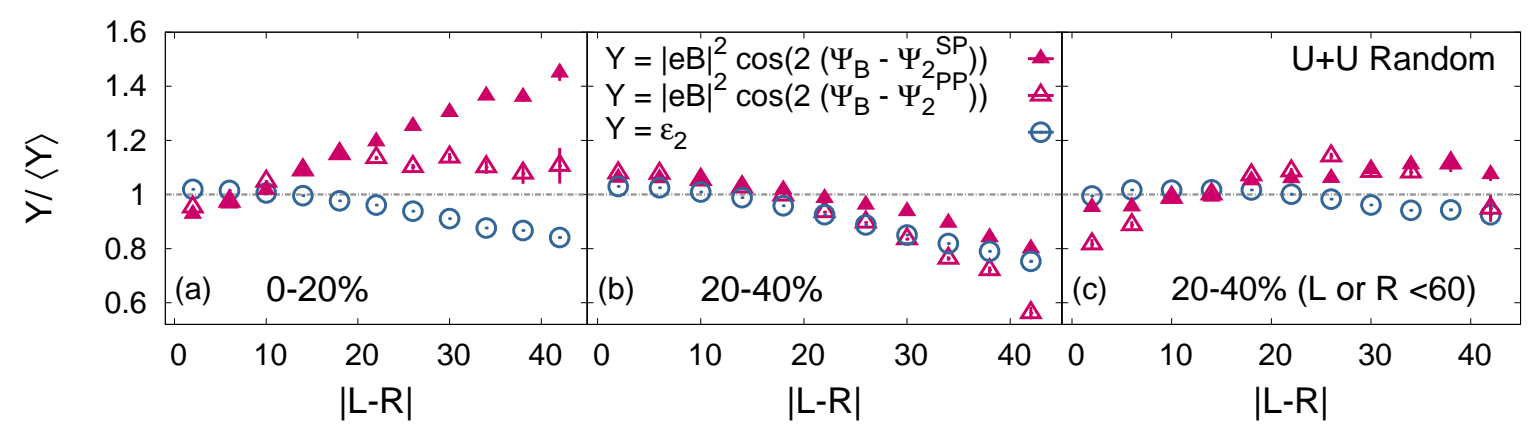

Figure 3: (Color online) The initial state $\varepsilon_{2}$ and $\gamma^{B}$ at the origin of the participant plane vs $|L-R|$ for different choices of $\psi_{R P}, \psi_{R P}=\psi_{2}^{P P}$ and $\psi_{2}^{S P}$. The plots are for $(0-20) \%$ and $(20-40) \%$ centrality cases. Panel (c) again shows the $(20-40) \%$ centrality class with additional cut on the maximum value of $L$ or $R$ [13].

by about $20 \%$. Interestingly, as shown in Fig. 3 (a), for the $(0-20) \%$ centrality bin $\gamma^{B}$ grows with increasing $|L-R|$. Thus we find that by varying $|L-R|$ alone one can in principle observe anti-correlation between the effects of $B$-field that lead to CME-like signals and flow-like effects. However we find that for the $(20-40) \%$ centrality bin, both $\varepsilon_{2}$ and $\gamma^{B}$ go down with increasing $|L-R|$. This is shown in Fig. 3 (b). This trend however drastically changes (as shown in Fig. 3 (c)) by restricting the number of spectators on any one side $(L$ or $R)$. We find that by applying a cut of $L$ or $R$ less than 60, once again we see a trend qualitatively similar to the $(0-20) \%$ bin.

In summary, we find that in $\mathrm{U}+\mathrm{U}$ collisions the spectator neutron asymmetry $|L-R|$ gives us a direct access to the initial state geometry and hence serves as a control parameter to trigger 
events with different values of initial anisotropy and disentangle flow background from the signals of CME.

\section{References}

[1] J. Liao, Anomalous transport effects and possible environmental symmetry "violation" in heavy ion collisions, Pramana 84, no. 5, 901 (2015) [arXiv:1401.2500 [hep-ph]].

[2] X. G. Huang, Electromagnetic fields and anomalous transports in heavy-ion collisions - A pedagogical review, [arXiv:1509.04073 [hep-ph]].

[3] D. E. Kharzeev, J. Liao, S. A. Voloshin and G. Wang, Chiral Magnetic Effect in High-Energy Nuclear Collisions - A Status Report, [arXiv:1511.04050 [hep-ph]].

[4] D. E. Kharzeev, Parity violation in hot QCD: why it can happen, and how to look for it, Phys.Lett. B633, 260-264, 2006, [arxiv: 0406125 [hep-ph] ]

[5] S. A. Voloshin, Phys.Rev. C70, 057901 (2004), [arXiv: hep-ph/0 406311 [hep-ph] ]

[6] L. Adamczyk et al.. (STAR Collaboration), Beam-energy dependence of charge separation along the magnetic field in Au+Au collisions at RHIC, Phys.Rev.Lett. 113, 052302 (2014), [arXiv:1404.1433[nucl-ex]]

[7] B. Abelev et al., (ALICE Collaboration), Charge separation relative to the reaction plane in $\mathrm{Pb}-\mathrm{Pb}$ collisions at $\sqrt{s_{\mathrm{NN}}}=2.76 \mathrm{TeV}$, Phys.Rev.Lett. 110, 012301 (2013), [arXiv:1207.0900[nucl-ex]]

[8] B. Abelev et al., (STAR Collaboration), Observation of charge-dependent azimuthal correlations and possible local strong parity violation in heavy ion collisions, Phys.Rev. C81, 054908 (2010), [arXiv:0909.1717 [nucl-ex]]

[9] B. Abelev et al., (STAR Collaboration),Azimuthal Charged-Particle Correlations and Possible Local Strong Parity Violation, Phys.Rev.Lett. 103, 251601 (2009), [arXiv:0909.1739 [nucl-ex]]

[10] A. Bzdak, V. Koch and J. Liao, Remarks on possible local parity violation in heavy ion collisions, Phys.Rev. C81, 031901 (2010), [arXiv:0912.5050 [nucl-th] ]

[11] S. A. Voloshin, Testing the Chiral Magnetic Effect with Central U+U collisions, Phys.Rev.Lett. 105, 172301 (2010), [arXiv:1006.1020 [nucl-th]]

[12] A. Bzdak, Suppression of elliptic-flow-induced correlations in an observable of possible local parity violation, Phys.Rev. C85, 044919 (2012), [arXiv:1112.4066 [nucl-th] ]

[13] S. Chatterjee and P. Tribedy, Separation of flow from the chiral magnetic effect in U $+U$ collisions using spectator asymmetry, Phys.Rev. C92, 1, 011902 (2015), [arXiv:1412.5103 [nucl-th]]

[14] J. Bloczynski, X. G. Huang, X. Zhang and J. Liao, Azimuthally fluctuating magnetic field and its impacts on observables in heavy-ion collisions, Phys.Lett. B718, 1529 (2013) [arXiv: 1209.6594 [nucl-th] ]

[15] V. Skokov, A. Y. Illarionov and V. Toneev, Estimate of the magnetic field strength in heavy-ion collisions, Int.J.Mod.Phys. A24, 5925 (2009) [arXiv:0907.1396 [nucl-th] ]

[16] G. Wang, (STAR Collaboration), Search for local parity violation with STAR ZDC-SMD, Journal of Physics: Conference Series 230, 012020 (2010) 